\title{
Fatores associados ao comprometimento da qualidade de vida em professores do
}

\section{Ensino Fundamental}

\author{
Factors associated with impaired quality of life of Elementary School teachers \\ Factores asociados al deterioro de la calidad de vida de los maestros de Primaria
}

Recebido: 01/10/2021 | Revisado: 09/10/2021 | Aceito: 19/10/2021 | Publicado: 20/10/2021

Ediálida Costa Santos

ORCID: https://orcid.org/0000-0001-7309-0092 Universidade Federal de Mato Grosso, Brasil E-mail: enf.edialida@gmail.com Mariano Martínez Espinosa

ORCID: https://orcid.org/0000-0002-0461-5673 Universidade Federal de Mato Grosso, Brasil E-mail: marianomphd@gmail.com

Samira Reschetti Marcon

ORCID: https://orcid.org/0000-0002-5191-3331 Universidade Federal de Mato Grosso, Brasil E-mail: samira.marcon@gmail.com

Léslie Piccolotto Ferreira

ORCID: https://orcid.org/0000-0002-3230-7248 Pontifícia Universidade Católica de São Paulo, Brasil E-mail: lesliepferreira@gmail.com

\begin{abstract}
Resumo
A qualidade de vida, saúde e trabalho dos professores constituem entre si complexas relações, contudo pesquisas com este tema são recentes e escassas. $\mathrm{O}$ objetivo deste estudo foi investigar fatores associados ao comprometimento dos domínios da qualidade de vida em professores do ensino fundamental de Cuiabá (MT). Realizou-se um estudo transversal, de amostragem probabilística, com 326 professores através de questionários padronizados autoaplicáveis. Os quatro domínios da escala de qualidade de vida, WHOQOL-bref (Físico, Psicológico, Relações sociais e Meio ambiente), foram analisados e considerado como comprometimento os indivíduos com escores $\leq$ mediana. Nas análises foram utilizadas a regressão de Poisson hierarquizada e os intervalos de confiança de $95 \%$. Os participantes tinham idade média de 43 anos e 87,12\% eram do sexo feminino. Os achados evidenciaram a associação do mesmo fator a um ou mais domínios avaliados, e associaram-se ao comprometimento dos domínios de qualidade de vida fatores como: estado civil, tempo de deslocamento para o trabalho, carga horária $\geq 40$ horas, falta de tempo na escola, percepção que o trabalho interfere na saúde, trabalho repetitivo, tempo de docência, esforço físico intenso, escola ruidosa, inatividade física, distúrbios no sono, falta de lazer, presença de transtorno mental comum, distúrbio de voz e queixas de sintomas osteomusculares.
\end{abstract}

Palavras-chave: Qualidade de vida; Saúde do trabalhador; Professores escolares; Promoção da saúde.

\begin{abstract}
The quality of life, health and work of teachers are complex relationships, however, research on this topic is recent and scarce. The aim of this study was to investigate factors associated with the impairment of quality of life domains in elementary school teachers in Cuiabá (MT). A cross-sectional, probabilistic sampling study was conducted with 326 teachers using standardized self-administered questionnaires. The four domains of the quality of life scale, WHOQOL-bref (Physical, Psychological, Social Relations and Environment), were analyzed and individuals with scores $\leq$ median were considered as impaired. The analyzes used hierarchical Poisson regression and $95 \%$ confidence intervals. The participants had an average age of 43 years and $87.12 \%$ were female. The findings showed the association of the same factor with one or more assessed domains, and associated with the impairment of quality of life domains such as: marital status, commuting time, workload $\geq 40$ hours, lack of time at school, perception that work interferes with health, repetitive work, teaching time, intense physical effort, ambient school noise, physical inactivity, sleep disorder, lack of leisure, presence of common mental disorder, voice disorder and complaints of musculoskeletal symptoms.
\end{abstract}

Keywords: Quality of life; Occupational health; School teachers; Health promotion.

\section{Resumen}

La calidad de vida, la salud y el trabajo de los maestros constituyen entre sí relaciones complejas, pero las investigaciones sobre este tema son recientes y escasas. El objetivo de este estudio fue investigar los factores 
asociados al deterioro de los dominios de la calidad de vida de los profesores de la enseñanza primaria en Cuiabá (MT). Se realizó un estudio transversal, de muestreo probabilístico, con 326 profesores mediante cuestionarios estandarizados autoaplicados. Los cuatro dominios de la escala de calidad de vida, WHOQOL-bref (Físico, Psicológico, Relaciones sociales y Medio ambiente), se analizaron y consideraron como compromiso a los individuos con escores $\leq$ mediana. En los análisis se utilizó la regresión jerárquica de Poisson y los intervalos de confianza del 95\%. Los participantes tenían una edad media de 43 años y el 87,12\% eran de sexo femenino. Los resultados mostraron la asociación de un mismo factor a uno o más dominios evaluados, y se asociaron con el deterioro de los dominios de calidad de vida factores como el estado civil, el tiempo de desplazamiento al trabajo, la carga de trabajo $\geq 40$ horas, la falta de tiempo en la escuela, la percepción de que el trabajo interfiere con la salud, el trabajo repetitivo, el tiempo de enseñanza, el esfuerzo físico intenso, la escuela ruidosa, la inactividad física, los trastornos del sueño, la falta de ocio, la presencia de un trastorno mental común, el trastorno de la voz y las quejas de síntomas musculoesqueléticos.

Palabras clave: Calidad de vida; Salud laboral; Maestros; Promoción de la salud.

\section{Introdução}

A relevância do professor para o ensino e desenvolvimento social é incontestável. Em que pese a sua importância, a literatura na atualidade tem destacado a existência de um quadro preocupante de adoecimento do professor em função de elementos relacionados ao trabalho (Scheuch, Haufe \& Seibt, 2015; Cortez et al., 2017; Luz et al., 2019; Graup et al.,2020). Ao professor cabe a realização de um conjunto de ações de ensino/aprendizagem dos alunos, relação com pares, formação/desenvolvimento profissional, de gestão e organização escolar. Tudo isso, faz com que o trabalho seja considerado complexo, o que exige dos professores equilíbrio (físico, psíquico, social e espiritual), refletindo desse modo na percepção de Qualidade de Vida (QV) (Davoglio et al., 2015).

Estudos têm demonstrado que o professor com frequência tem sido acometido por problemas ocupacionais relacionados à ocorrência de distúrbios de voz (Cortez et al.,2017; Luz et al.,2019; Mota et al., 2019), de transtornos mentais, como o estresse e Burnout (Tabeleão, Tomasi \& Neves, 2011; Koga et al., 2015; Cortez et al., 2017), e de dor osteomusculares (Fernandes, Rocha \& Fagundes, 2011; Scheuch, Haufe \& Seibt, 2015; Cebalhos \& Santos, 2015). Os relatos sobre a percepção de que ocorre uma invasão multiforme da vida pelo trabalho (Silva \& Fisher, 2020), a ocorrência de problemas relacionados ao ambiente, organização e condições de trabalho, têm afetado por vezes a saúde do professor (Luz et al.,2019). Neste contexto, nota-se que a saúde, o trabalho e a QV possuem estreitas relações.

Convém destacar que a Organização Mundial de Saúde (OMS) define a QV como sendo a "percepção do indivíduo sobre sua posição na vida, no contexto da cultura e dos sistemas de valores nos quais ele vive, e em relação a seus objetivos, expectativas, padrões e preocupações" (WHO, 2012). Assim, a OMS percebe que a qualidade de vida é um conceito subjetivo, multidimensional e passível de mensuração pelo instrumento World Health Organization Quality of Life (WHOQOL), que fornece medidas genéricas de QV e é considerado apropriado para estudos epidemiológicos, de planejamento e avaliação de sistemas de saúde (Minayo, Hartz \& Buss, 2000).

A avaliação da QV por instrumentos padronizados é vasta na literatura, em especial para grupos de pessoas com agravos específicos e idosos, contudo essas produções com grupos de trabalhadores, como os professores, são recentes e escassas. Em pesquisas com professores, por exemplo, foram citados como fatores associados à QV elementos como o desgaste profissional caracterizado pela síndrome de Burnout (Tabeleão, Tomasi \& Neves, 2011), sintomatologia osteomuscular (Fernandes, Rocha \& Fagundes, 2011), tempo de magistério e carga horária semanal (Pereira, Teixeira \& Lopes, 2013; Pereira et al., 2014), presença de zumbido, intolerância a sons intensos, ansiedade e cefaleia (Pimentel et al.,2016) e a classe econômica (Tavares et al., 2015).

Por tudo o que foi exposto, verifica-se que fatores sociodemográficos, ocupacionais, hábitos de vida e sono, transtornos mentais comuns, distúrbios de voz e queixas osteomusculares podem influenciar na QV. Desse modo, ações de reabilitação, prevenção e promoção da saúde poderiam ser propostas a partir do reconhecimento desses fatores intervenientes 
na QV. Assim, o objetivo principal do presente estudo foi analisar a percepção de QV e investigar os fatores associados ao comprometimento dos domínios da qualidade de vida em professores do ensino fundamental.

\section{Metodologia}

Trata-se de um estudo epidemiológico transversal e analítico, desenvolvido com professores do ensino fundamental da rede pública municipal da capital Cuiabá- Mato Grosso, Brasil. Para o planejamento amostral da pesquisa utilizou-se amostragem probabilística simples, estratificada e sistemática (Bolfarine \& Bussab, 2005). No cálculo do tamanho da amostra, considerou-se uma prevalência de 50\% para desfecho desconhecido, um intervalo de confiança de $95 \%$, um erro amostral de 5\% e uma população de 1317 professores, estimada no Censo Educacional (INEP, 2018). Assim, o tamanho amostral mínimo necessário era de 298 participantes. Com um acréscimo de 35\% para perdas, tendo o intuito de assegurar a representatividade da amostra e considerando elevada possibilidade de recusa segundo experiência de estudo anterior com metodologia semelhante (Pereira, Teixeira \& Lopes, 2013), a amostra final totalizou aproximadamente 403 professores.

O método de amostragem probabilística estratificada e sistemática foi empregado neste estudo, tendo considerado uma divisão de participantes proporcional ao tamanho dos estratos (regiões administrativas do município: Norte, Sul, Leste, Oeste) e para o sorteio das escolas, de modo aleatório e sistemático considerou o fator de repetição 4 . Dentre as 75 escolas municipais, foram selecionadas aleatoriamente 21 escolas e os professores participantes.

Como critério de inclusão foi considerado no estudo todos os professores que estavam em pleno exercício profissional e excluídos aqueles em desvio de função, de licença ou afastados da sala de aula no momento da coleta de dados. Assim, aos professores foram entregues o questionário para a coleta de dados, com a devolução prevista para o dia posterior ou em até duas tentativas de recolhimento. O período da coleta de dados ocorreu entre os meses de setembro a dezembro de 2017.

O questionário autoaplicado constou de um compilado de instrumentos internacionais e previamente validados no Brasil: World Health Organization Quality of Life instrument (WHOQOL-BREF)(WHO, 2012); Nordic Musculoskeletal Questionnaire (NMQ) (Pinheiro, Tróccoli \& Carvalho, 2002); Self-Reporting Questionnaire (SRQ-20) (Gonçalves, Stein \& Kapczinski, 2008); e por instrumentos construídos e validados nacionalmente: Condição de Produção Vocal do Professor (CPV-P) e Índice de Triagem de Distúrbio de Voz - (ITDV) (Ghirardi et al., 2013; Giannini, Latorre \& Ferreira, 2016), além de algumas questões adicionais sobre aspectos socioeconômicos e hábitos e estilo de vida que não estavam contemplados nos outros instrumentos e que poderiam influenciar na qualidade de vida.

Os desfechos foram os quatro domínios (Físico, Psicológico, Relações sociais e Meio ambiente) mensurados pelo WHOQOL-Bref (WHO, 2012). Esse instrumento avalia a QV a partir de 26 questões, sendo dois itens analisados separadamente sobre a autoavaliação da satisfação com a saúde e percepção de qualidade de vida, e as demais 24 questões distribuídas entre os quatro domínios, que em sintaxe proposta pelo Grupo WHOQOL (WHO, 2012) são transformadas em escores numa escala de zero (pior condição) a 100 (melhor condição).

Desse modo, os desfechos avaliados foram: a) domínio Físico, que incluem facetas que avaliam a dor e desconforto; energia/fadiga, sono/repouso, mobilidade, atividades da vida cotidiana, dependência de medicação/tratamentos e capacidade para o trabalho; b) domínio Psicológico: abrange itens relativos a sentimentos positivos, pensamento, aprendizagem, memória e concentração, autoestima, imagem corporal/aparência, sentimentos negativos, espiritualidade, religiosidade e crenças pessoais; c) domínio Relações sociais, incluem facetas relacionadas a relações pessoais, suporte social e atividade sexual; e d) domínio Meio ambiente, com facetas relacionadas a segurança física e proteção, ambiente no lar, recursos financeiros, cuidados de saúde e sociais, oportunidade de adquirir novas informações e habilidades, participação em oportunidades de recreação/lazer, ambiente físico (poluição, ruído, trânsito, clima) e transporte (WHO, 2012). 
As prevalências dos desfechos foram obtidas das categorizações dos escores em dois grupos, considerando como ponto de corte a mediana, uma vez que a distribuição dos escores neste estudo apresentou distribuição assimétrica para todos os domínios, sendo considerados como grupo de maior comprometimento os indivíduos com escore igual ou abaixo do valor da mediana (Espinosa et al., 2019). Para isto foram determinados como pontos de corte a mediana, equivalendo a 53,13 para o domínio Meio ambiente, de 66,67 para Relações sociais, de 67,86 para o Físico e de 70,83 para o Psicológico. Assim, nestes casos a categorização a partir dos valores da mediana podem produzir categorias representativas de uma pior e melhor qualidade de vida (Espinosa et al., 2019).

Quanto as variáveis independentes, essas foram agrupadas em quatro Blocos, sendo:

Bloco 1 - Características sociodemográficas: a idade foi categorizada em duas categorias sendo uma com valores iguais ou abaixo do valor da media ( $\leq 43,01$ anos), e outra com idade superior a média ( $>43,01$ anos); sexo (feminino ou masculino); estado civil (solteiro/separado/desquitado/viúvo ou casado/qualquer forma de união); escolaridade (graduação ou pósgraduação); tempo de viagem de casa para o trabalho (em categorias, <15 minutos, 16 - 30 minutos, 31 - 60 minutos e mais de 60minutos).

Bloco 2 - Características do trabalho docente

2.1 - Situação funcional: tempo de profissão ( $\geq 20$ anos, 10-19 anos e $\leq 9$ anos); quantidade de escolas de trabalho (em 1 escola, 2 ou mais escolas); carga horária em contato com os alunos ( $\geq 40$ horas, 21-40h, $\leq 20$ horas); e vínculo empregatício (Contrato temporário/Substituto ou Efetivo/com classe definida).

2.2 - Ambiente de trabalho: Presença de ruídos; Acústica satisfatória; Presença de eco; Presença de poeira; Presença de fumaça; Temperatura agradável; Umidade do ar adequada; Iluminação adequada; Limpeza satisfatória na escola; Higiene adequada nos banheiros; Tamanho da sala adequado; Móveis adequados à estatura.

2.3 - Organização do trabalho: local adequado para descanso do professor; Liberdade planejamento e realização de atividades; Supervisão constante; Ritmo estressante; Material adequado; Material Suficiente; Trabalho monótono; Trabalho repetitivo; Tempo para realizar as atividades na escola; Leva trabalho para casa; Facilidade para ausentar-se da sala; Esforço físico intenso; Carrega peso com frequência; Comprometimento dos funcionários; Satisfação na função; Estresse no trabalho; e Percepção de que o trabalho interfere na saúde. As situações de violência que presenciam na escola: Roubo de objetos pessoais; Roubo de materiais da escola; Manifestação de bulling; Briga entre alunos; Violência contra professores e funcionários; Atos de vandalismo contra o prédio; Violência a porta da escola.

Bloco 3 - Características dos hábitos de vida e sono: os participantes foram investigados sobre Atividades de Lazer; Fumo; Uso de bebida alcoólica; Alimentação em horários regulares; Realiza atividade física ou esporte; Acorda durante a noite; Acorda descansado; Horas de Sono (categorizado em $\leq 6$ horas e $\geq 7$ horas).

As respostas referentes aos itens do Bloco 2 (Ambiente de trabalho e Organização do trabalho) e do Bloco 3 foram dicotomizadas em "às vezes/sempre" e "nunca/raramente" para fins de análise, com exceção de horas de sono.

Bloco 4 - Condições de saúde: foram avaliados aspectos relacionados à ocorrência de distúrbio de voz, transtorno mental comum e distúrbios osteomusculares por três instrumentos validados. O instrumento ITDV (Ghirardi et al.,2013), investigou o distúrbio de voz a partir do relato da ocorrência de 12 sintomas vocais, sendo considerado como a presença de distúrbios de voz para quem referiu "as vezes ou sempre" à cinco ou mais itens. O instrumento SQR-20, proposto pela Organização Mundial de Saúde, avaliou a ocorrência de sintomas de Transtorno Mental Comum (TMC) a partir de 20 perguntas, sendo adotado o 
ponto de corte para quem respondeu "sim" em $\geq 8$ pontos (presente) (Gonçalves, Stein \& Kapczinski, 2008). No instrumento NMQ, os participantes relatam "sim" ou "não" para a ocorrência de sintomas osteomusculares em uma figura anatômica, avaliada nos 12 últimos meses e nos últimos 7 dias para quem respondeu "sim" independentemente da quantidade de vezes e do segmento corporal indicado (Pinheiro, Tróccoli \& Carvalho, 2002).

Para as análises, as variáveis foram descritas por meio de frequências absolutas e relativas. Nas razões de prevalência brutas e ajustadas foram considerados ajustes robustos para a variância. Nesse último caso foi obtido por um modelo de regressão múltipla de Poisson hierarquizado em blocos, para analisar a relação dos fatores associados ao desfecho (Victora et al., 1997). Assim, foram incluídas na regressão as variáveis que apresentaram $p \leq 0,20$ nas análises brutas.

As variáveis foram ajustadas entre si em cada bloco e para as de nível superior, assim, inicialmente, ingressaram as variáveis do bloco 1 , que se juntaram às do bloco 2 , após o bloco 3 e, por último, o bloco 4 . Foram mantidas no modelo final para ajuste e controle de confusão àquelas com $\mathrm{p} \leq 0,20$ e consideradas estatisticamente significantes associadas ao desfecho as variáveis com $\mathrm{p}<0,05$.

Nesta pesquisa os dados foram duplamente digitados e comparados no programa EpiInfo para corrigir eventuais erros de digitação. As análises estatísticas foram realizadas no programa SPSS 20.

As diretrizes éticas foram atendidas com a aprovação por Comitê de Ética em Pesquisa do Hospital Júlio Muller (CAAE: 59503916.7.0000.5541) e todos os participantes receberam esclarecimentos e concordaram assinando o Termo de Consentimento Livre e Esclarecido.

\section{Resultados}

O questionário autoaplicável foi entregue para 403 professores e desses foram considerados válidos 326 (80,90\% da amostra prevista). Um total de $17,62 \%$ não devolveu o questionário após três tentativas de contato e houve perda de 1,48\% de questionários incompletos.

A maioria da amostra participante era do sexo feminino (87,12\%), com idade inferior a 43 anos $(50,33 \%$ - idade média de 43,01 anos, DP=9,31 anos), de escolaridade no nível de pós-graduação $(74,23 \%)$ e conviviam com cônjuge $(62,70 \%)$. Dos aspectos profissionais, grande parte trabalhava como docente por $\leq 9$ anos (38,94\%) e por seguinte,31,78\% tinham tempo igual e superior a 20 anos no ensino. A maioria lecionava em uma única escola $(58,95 \%)$, com carga horária entre 21-40 horas semanais com alunos (45,28\%) e tinham o vínculo empregatício como contratado temporário (54,60\%).

A prevalência de comprometimento da qualidade de vida nos professores foi de 57,85\% no domínio Físico (IC95\%:0,52-0,63), de 55,52\% no Relação social (IC95\%:0,50-0,61), de 51,90\% no domínio Psicológico (IC95\%:0,46-0,58), e de 47,85\% no Meio ambiente (IC95\%: 0,42-0,53).

O modelo de regressão confirmou a associação do comprometimento do domínio Físico com fatores como a carga horária $\geq 40$ horas, nunca/raramente terem tempo para realizar as atividades na escola, a percepção de que às vezes/sempre o trabalho interfere na saúde, nunca/raramente realizam atividade física ou esportes, a presença de transtorno mental comum e distúrbios osteomusculares nos últimos 7 dias (Tabela 1). 
Tabela 1 - Razões de prevalência brutas e ajustadas dos fatores associados ao comprometimento do domínio Físico da qualidade de vida em professores do ensino fundamental.

\begin{tabular}{|c|c|c|c|c|c|c|c|}
\hline Bloco & Variáveis & $\begin{array}{l}\mathbf{R P} \\
\text { bruta }\end{array}$ & IC 95\% & $p$ & RP ajustada & IC 95\% & $p$ \\
\hline \multirow[t]{10}{*}{2} & Carga horária & & & & & & \\
\hline & $\geq 40$ horas & 1,29 & $1,04-1,60$ & 0,021 & 1,32 & $1,04-1,68$ & $0,022^{*}$ \\
\hline & $21-40 \mathrm{~h}$ & 1,07 & $0,89-1,29$ & 0,460 & 1,14 & $0,94-1,38$ & 0,176 \\
\hline & $\leq 20$ horas & 1,00 & & & 1,00 & & \\
\hline & $\begin{array}{l}\text { Tempo para realizar as } \\
\text { atividades na escola }\end{array}$ & & & & & & \\
\hline & Nunca/raramente & 1,60 & $1,36-1,90$ & $<0,001$ & 1,27 & $1,08-1,49$ & $0,003^{*}$ \\
\hline & Às vezes/sempre & 1,00 & & & 1,00 & & \\
\hline & Trabalho interfere na saúde & & & & & & \\
\hline & Às vezes/sempre & 2,11 & $1,52-2,94$ & $<0,001$ & 1,59 & $1,12-2,26$ & $0,009^{*}$ \\
\hline & Nunca/raramente & 1,00 & & & 1,00 & & \\
\hline \multirow[t]{6}{*}{3} & Atividade física ou esportes & & & & & & \\
\hline & Nunca/raramente & 1,41 & $1,16-1,72$ & 0,001 & 1,23 & $1,02-1,49$ & $0,029^{*}$ \\
\hline & Às vezes/sempre & 1,00 & & & 1,00 & & \\
\hline & Sono: acorda descansado & & & & & & \\
\hline & Nunca/raramente & 1,54 & $1,30-1,83$ & $<0,001$ & 1,13 & $0,95-1,33$ & 0,155 \\
\hline & Às vezes/sempre & 1,00 & & & 1,00 & & \\
\hline \multirow[t]{12}{*}{4} & Transtornos Mentais comuns & & & & & & \\
\hline & Presente & 2,04 & $1,72-2,38$ & $<0,001$ & 1,23 & $1,01-1,50$ & $0,036^{*}$ \\
\hline & Ausente & 1,00 & & & 1,00 & & \\
\hline & Distúrbios de voz & & & & & & \\
\hline & Presente & 1,60 & $1,31-1,98$ & $<0,001$ & 1,21 & $0,97-1,50$ & 0,088 \\
\hline & Ausente & 1,00 & & & 1,00 & & \\
\hline & $\begin{array}{l}\text { Sintomas osteomuscular nos } 12 \\
\text { últimos meses }\end{array}$ & & & & & & \\
\hline & Sim & 1,88 & $1,37-2,59$ & $<0,001$ & 1,43 & $0,99-2,05$ & 0,052 \\
\hline & Não & 1,00 & & & 1,00 & & \\
\hline & $\begin{array}{l}\text { Sintomas osteomuscular } \\
\text { nos últimos } 7 \text { dias }\end{array}$ & & & & & & \\
\hline & Sim & 1,88 & $1,53-2,31$ & $<0,001$ & 1,38 & $1,09-1,75$ & $0,007^{*}$ \\
\hline & Não & 1,00 & & & 1,00 & & \\
\hline
\end{tabular}

Nota: As variáveis: Sono, Distúrbios de voz e Sintomas osteomusculares nos 12 últimos meses, embora não estatisticamente significativa permaneceram no modelo como variável de ajuste $(\mathrm{p}<0,20)$. *:variável estatisticamente significante ao nível de 0,05 .

Fonte: Autores.

Quanto ao comprometimento do domínio Psicológico, foram fatores associados à manifestação de que o trabalho é repetitivo às vezes/sempre; nunca/raramente possuem tempo para realizar as atividades na escola; que o trabalho interfere na saúde; dificuldades quanto ao sono, manifesta pelo relato de que às vezes/sempre acordam durante a noite; e a presença de transtornos mentais comuns. Neste domínio, a ocorrência de transtorno mental registrou maior prevalência (RP: 1,81; $\mathrm{p}<0,001$ ), e foi mantida a variável sexo para o ajuste das demais por plausibilidade teórica (Tabela 2). 
Tabela 2 - Razões de prevalência brutas e ajustadas dos fatores associados ao comprometimento do domínio Psicológico da qualidade de vida em professores do ensino fundamental.

\begin{tabular}{|c|c|c|c|c|c|c|c|}
\hline Bloco & Variáveis & $\begin{array}{l}\text { RP } \\
\text { bruta }\end{array}$ & IC $95 \%$ & $p$ & $\begin{array}{l}\text { RP } \\
\text { ajustada }\end{array}$ & IC 95\% & $p$ \\
\hline \multirow[t]{3}{*}{1} & Sexo & & & & & & \\
\hline & Feminino & 1,14 & $0,93-2,12$ & 0,108 & 1,24 & $0,81-1,90$ & 0,328 \\
\hline & Masculino & 1,00 & & & 1,00 & & \\
\hline \multirow[t]{9}{*}{2} & Trabalho repetitivo & & & & & & \\
\hline & Às vezes/sempre & 1,67 & $1,31-2,12$ & $<0,001$ & 1,35 & $1,08-1,69$ & $0,007^{*}$ \\
\hline & Nunca/raramente & 1,00 & & & 1,00 & & \\
\hline & $\begin{array}{l}\text { Tempo para realizar as atividades na } \\
\text { escola }\end{array}$ & & & & & & \\
\hline & Nunca/raramente & 1,54 & $1,22-1,94$ & $<0,001$ & 1,25 & $1,03-1,53$ & $0,024^{*}$ \\
\hline & Às vezes/sempre & 1,00 & & & 1,00 & & \\
\hline & Trabalho interfere na saúde & & & & & & \\
\hline & Às vezes/sempre & 2,30 & $1,54-3,45$ & $<0,001$ & 1,59 & $1,07-2,37$ & $0,022^{*}$ \\
\hline & Nunca/raramente & 1,00 & & & 1,00 & & \\
\hline \multirow[t]{3}{*}{3} & Sono: Acorda durante a noite & & & & & & \\
\hline & Às vezes/sempre & 1,89 & $1,31-2,73$ & 0,001 & 1,44 & $1,02-2,02$ & $0,038^{*}$ \\
\hline & Nunca/raramente & 1,00 & & & 1,00 & & \\
\hline \multirow[t]{3}{*}{4} & Transtornos Mentais comuns & & & & & & \\
\hline & Presente & 2,40 & $1,95-2,95$ & $<0,001$ & 1,81 & $1,46-2,26$ & $<0,001^{*}$ \\
\hline & Ausente & 1,00 & & & 1,00 & & \\
\hline
\end{tabular}

Nota: A variável "Sexo" permanece como ajuste ao modelo por plausibilidade teórica, embora não estatisticamente significativa. ":variável estatisticamente significante ao nível de 0,05 .

Fonte: Autores.

O comprometimento no domínio de Relações sociais esteve associado à ocorrência de fatores como estado civil sem o cônjuge (RP: 1,21; p=0,045), quem atuou entre 10-19 anos como docentes (RP: 1,36; p=0,006), percebeu no ambiente de trabalho da escola a ocorrência de ruído às vezes/sempre (RP: 1,48; $p=0,016)$, realizou esforço físico intenso no trabalho às vezes/sempre (RP: 1,$23 ; p=0,032$ ), presença de transtornos mentais comuns (RP: 1,$35 ; p=0,004$ ); e queixa osteomuscular nos últimos 12 meses (RP: 1,52; $p=0,018$ ) (Tabela 3$)$. 
Tabela 3 - Razões de prevalência brutas e ajustadas dos fatores associados ao comprometimento do domínio Relações sociais da qualidade de vida em professores do ensino fundamental.

\begin{tabular}{|c|c|c|c|c|c|c|c|}
\hline Bloco & Variáveis & RP bruta & IC $95 \%$ & $p$ & $\begin{array}{l}\text { RP } \\
\text { ajustada }\end{array}$ & IC 95\% & $\boldsymbol{P}$ \\
\hline \multirow[t]{3}{*}{1} & Estado Civil & & & & & & \\
\hline & Sem cônjuge & 1,16 & $0,95-1,42$ & 0,132 & 1,21 & $1,00-1,47$ & $0,045^{*}$ \\
\hline & Com cônjuge & 1,00 & & & 1,00 & & \\
\hline \multirow[t]{10}{*}{2} & Tempo de docência & & & & & & \\
\hline & $\geq 20$ anos & 0,98 & $0,80-1,22$ & 0,893 & 1,07 & $0,84-1,37$ & 0,591 \\
\hline & $10-19$ anos & 1,26 & $1,04-1,53$ & 0,021 & 1,36 & $1,09-1,70$ & $0,006^{*}$ \\
\hline & $\leq 9$ anos & 1,00 & & & 1,00 & & \\
\hline & Escola ruidosa & & & & & & \\
\hline & Às vezes/sempre & 1,63 & $1,17-2,28$ & 0,004 & 1,48 & $1,07-2,04$ & $0,016^{*}$ \\
\hline & Nunca/raramente & 1,00 & & & 1,00 & & \\
\hline & $\begin{array}{l}\text { Realiza esforço físico intenso no } \\
\text { trabalho }\end{array}$ & & & & & & \\
\hline & Às vezes/sempre & 1,38 & $1,14-1,67$ & 0,001 & 1,23 & $1,02-1,49$ & $0,032^{*}$ \\
\hline & Nunca/raramente & 1,00 & & & 1,00 & & \\
\hline \multirow[t]{9}{*}{4} & Transtornos mentais comuns & & & & & & \\
\hline & Presente & 1,71 & $1,43-2,04$ & $<0,001$ & 1,35 & $1,10-1,66$ & $0,004^{*}$ \\
\hline & Ausente & 1,00 & & & 1,00 & & \\
\hline & Distúrbios de voz & & & & & & \\
\hline & Presente & 1,50 & $1,22-1,85$ & $<0,001$ & 1,21 & $0,96-1,53$ & 0,112 \\
\hline & Ausente & 1,00 & & & 1,00 & & \\
\hline & $\begin{array}{l}\text { Sintomas osteomuscular nos } 12 \\
\text { últimos meses }\end{array}$ & & & & & & \\
\hline & Sim & 1,72 & $1,26-2,35$ & 0,001 & 1,52 & $1,07-2,14$ & $0,018^{*}$ \\
\hline & Não & 1,00 & & & 1,00 & & \\
\hline
\end{tabular}

Nota: A variável de "Distúrbios de voz", embora não estatisticamente significativa permaneceu no modelo como variável de ajuste ( $\mathrm{p}<0,20)$. *:variável estatisticamente significante ao nível de 0,05 .

Fonte: Autores.

No domínio Meio ambiente, o modelo de regressão confirma a associação significativa do comprometimento à fatores como tempo de deslocamento da casa para trabalho ser $\geq 31$ minutos; à percepção do professor que o trabalho interfere na saúde; nunca/raramente realizam atividades de lazer; baixa qualidade do sono, investigado pela condição de nunca/raramente acordam descansados; a presença do distúrbio de voz; e ocorrência de queixas osteomusculares nos últimos 7 dias (Tabela 4). 
Tabela 4 - Razões de prevalência brutas e ajustadas dos fatores associados ao comprometimento do domínio Meio ambiente da qualidade de vida em professores do ensino fundamental.

\begin{tabular}{|c|c|c|c|c|c|c|c|}
\hline Bloco & Variáveis & RP bruta & IC $95 \%$ & $p$ & $\begin{array}{l}\text { RP } \\
\text { ajustada }\end{array}$ & IC 95\% & $\boldsymbol{P}$ \\
\hline \multirow[t]{4}{*}{1} & $\begin{array}{l}\text { Tempo deslocamento da casa para } \\
\text { trabalho }\end{array}$ & & & & & & \\
\hline & $\geq 31 \mathrm{~min}$ & 1,49 & $1,17-1,91$ & 0,001 & 1,60 & $1,25-2,06$ & $<0,001^{*}$ \\
\hline & $16-30 \mathrm{~min}$. & 1,10 & $0,86-1,40$ & 0,463 & 1,19 & $0,91-1,54$ & 0,202 \\
\hline & $\leq 15 \mathrm{~min}$ & 1,00 & & & 1,00 & & \\
\hline \multirow[t]{6}{*}{2} & Material de trabalho adequado & & & & & & \\
\hline & Nunca/raramente & 1,50 & $1,18-1,91$ & 0,001 & 1,22 & $0,98-1,52$ & 0,081 \\
\hline & Às vezes/sempre & 1,00 & & & 1,00 & & \\
\hline & Trabalho interfere na saúde & & & & & & \\
\hline & Às vezes/sempre & 1,85 & $1,28-2,66$ & 0,001 & 1,43 & $1,00-2,05$ & $0,047^{*}$ \\
\hline & Nunca/raramente & 1,00 & & & 1,00 & & \\
\hline \multirow[t]{6}{*}{3} & Atividades de lazer & & & & & & \\
\hline & Nunca/raramente & 1,71 & $1,38-2,13$ & $<0,001$ & 1,58 & $1,27-1,95$ & $<0,001^{*}$ \\
\hline & Às vezes/sempre & 1,00 & & & 1,00 & & \\
\hline & Sono: acorda descansado & & & & & & \\
\hline & Nunca/raramente & 1,85 & $1,49-2,29$ & $<0,001$ & 1,42 & $1,14-1,77$ & $0,002^{*}$ \\
\hline & Às vezes/sempre & 1,00 & & & 1,00 & & \\
\hline \multirow[t]{7}{*}{4} & Distúrbios de voz & & & & & & \\
\hline & Presente & 1,73 & $1,33-2,26$ & $<0,001$ & 1,30 & $1,01-1,69$ & $0,044^{*}$ \\
\hline & Ausente & 1,00 & & & 1,00 & & \\
\hline & Sintomas osteomuscular nos últimos & & & & & & \\
\hline & 7 dias & & & & & & \\
\hline & Sim & 1,67 & $1,30-2,14$ & $<0,001$ & 1,47 & $1,15-1,87$ & $0,002^{*}$ \\
\hline & Não & 1,00 & & & 1,00 & & \\
\hline
\end{tabular}

Nota: A variável “material de trabalho adequado", embora não estatisticamente significativa permaneceu no modelo como variável de ajuste (p<0,20). *:variável estatisticamente significante ao nível de 0,05 .

Fonte: Autores.

\section{Discussão}

A qualidade de vida é um conceito multidimensional e subjetivo. No presente estudo, vários fatores se associaram ao comprometimento dos domínios Físico, Psicológico, Relações sociais e Meio ambiente. Assim, os achados evidenciaram a associação do mesmo fator a um ou mais domínios avaliados reforçando a inter-relação existente entre fatores que afetam a QV.

O perfil sociodemográfico dos professores assemelha-se ao encontrado em outras pesquisas com amostras representativas da categoria profissional (Tabeleão, Tomasi \& Neves, 2011; Fernandes, Rocha \& Fagundes, 2011). Convém destacar que no ensino fundamental a maioria dos professores é do sexo feminino, e isso pode estar relacionado a influência histórica do processo de inserção da mulher no mercado formal de trabalho em profissões que guardam similaridades com a esfera doméstica de cuidado e criação dos filhos (Neves, Brito \& Muniz, 2019). No estudo em questão, para os modelos finais, as diferenças quanto ao sexo não permaneceram associadas, contudo, no domínio Psicológico a variável foi mantida como ajuste por plausibilidade teórica. Nesse aspecto, foram considerados estudos que indicaram que a variável sexo tem influenciado principalmente os aspectos psicológicos e de sofrimento mental de professoras (Pereira et al., 2014; Tostes et al.,2018; Neves, Brito \& Muniz, 2019).

Dos fatores sociodemográficos (Bloco 1) que comprometeram a QV, o estado civil (sem cônjuge) esteve associado ao domínio Relações sociais. Similarmente, em um município do sul do Brasil foi encontrado que professores que viviam sem companheiros obtiveram menores médias no domínio Relações sociais (Folle \& Farias, 2012). Nota-se que essa associação 
pode ser esperada, uma vez se encontram nesse domínio facetas relacionadas a relações pessoais, suporte social e atividade sexual.

Ainda no Bloco 1, o tempo de deslocamento para o trabalho maior que 30 minutos comprometeu o domínio Meio ambiente. Constata-se que essa é uma variável pouco investigada entre os estudos que envolvem professores, contudo é notório que a demora no deslocamento pode ensejar a perda de tempo livre que poderia ser utilizado para saúde e bem-estar e também perdas na produtividade (Pero \& Stefanelli, 2015). Entre professores franceses no início de carreira foram observados menores escores no domínio meio ambiente, e esse grupo geralmente exibia o tempo de viagem em média semelhante ao registrado nesta pesquisa, além de terem informado trabalhar sob forma de contrato, em mais de um estabelecimento ou nos subúrbios e com alunos de menor classe social (Zavidovique, Gilbert \& Vercambre-Jacquot, 2018). Nesse sentido, alocar professores em escolas próximas à residência pode ser uma alternativa para melhorar a qualidade de vida, entretanto outros aspectos relacionados ao domínio Meio ambiente como trabalho e a renda também devem ser analisados.

Das características referentes ao trabalho docente (Bloco 2), a percepção dos professores de que há interferência desse na própria saúde se destaca visto que esteve associada aos domínios Físico, Psicológico e Meio ambiente. A noção de que os agravos advindos do trabalho se projetam sobre a vida pessoal de professores foi relatada em outro estudo (Silva \& Fischer, 2020), bem como também foi registrada a percepção negativa quanto à falta de equilíbrio entre a vida pessoal e profissional, como aspectos que afetam os professores (Filis et al.,2016; Birolim et al., 2019). Tais percepções reforçam a necessidade de se discutir os elementos relacionados ao trabalho que repercutem na saúde e qualidade de vida dos professores.

Os fatores como carga horária $\geq 40$ horas e a falta de tempo para realizar as atividades na escola encontram-se associados ao comprometimento do domínio Físico e esse último fator esteve também associado ao domínio Psicológico. A influência da elevada carga horária sobre o domínio Físico também foi relatada em outras pesquisas (Pereira, Teixeira \& Lopes, 2013; Pereira et al.,2014), e associada ao trabalho de alta exigência (Birolim et al.,2019). Ao professor cabe além das atividades de ensino, várias tarefas como, atendimento ao aluno e controle da turma, preparo de aulas, elaboração e correção de provas/trabalhos, preenchimento de múltiplos instrumentos e formulários de controle. Nota-se que tanto a intensificação do trabalho, bem como as situações de sobreposição de tarefas podem explicar o cansaço físico, vocal e mental do professor (Assunção \& Oliveira, 2009). Desse modo, é recomendável que as políticas públicas considerem a carga horária e a organização das atividades dentro da jornada de trabalho como elementos para a promoção da saúde dos professores.

Os fatores como o tempo de docência entre 10-19 anos, mencionar a presença de ruídos na escola e realizar esforço físico intenso no trabalho, constantes do Bloco 2 e que se referem a aspectos relacionados ao trabalho, se associaram ao comprometimento do domínio Relações sociais. Na literatura há menção referente ao domínio Relações sociais e a tendência a escores inferiores na medida em que aumentaram os anos trabalhados (Pereira et al., 2014). Outros fatores como a percepção de zumbido, intolerância a sons intensos, ansiedade e cefaleia estiveram relacionados à pior qualidade de vida (Pimentel et al.,2016). Esses achados são plausíveis, se considerarmos que o cansaço físico e mental decorrente do trabalho pode interferir na vida pessoal dos professores, inclusive nas relações sociais.

Perceber o trabalho como sendo repetitivo foi fator associado ao comprometimento do domínio Psicológico. De modo análogo, estudo indicou que a percepção de que o trabalho é repetitivo associou-se à ocorrência de transtorno mental entre professores da rede privada (Delcor et al, 2004). Esse achado pode ser considerado preocupante, pois a repetitividade no trabalho pode predispor a déficits de criatividade e motivação refletindo no comprometimento psicológico.

Dentre os fatores relacionados aos hábitos de vida e sono (Bloco 3), a não realização de esporte ou atividade física esteve associado ao comprometimento do domínio Físico. Os benefícios da prática de atividade física ficaram demonstrados em pesquisa nos quais professores fisicamente ativos percebiam melhor a própria saúde quando comparados aos inativos (Santos \& Marques, 2013), contudo, dados da literatura convergem em afirmar que é elevado o número de professores que não 
realizam atividades físicas (Folle \& Farias, 2012; Dias et al., 2017). A atividade física insuficiente nem sempre está atrelada a uma decisão e disposição individual, mas elementos vinculados diretamente com a ocupação do professor podem interferir. Desse modo, estudo com professores evidenciou a associação entre atividade física insuficiente com a percepção ruim ou regular de equilíbrio entre vida pessoal e profissional, tempo de permanência em pé afetando o trabalho, baixa ou muito baixa capacidade atual para as exigências físicas do trabalho entre outros (Dias et al., 2017). Os achados da presente pesquisa corroboram esses estudos e indicam a possibilidade de ações que possam ser instituídas no ambiente de trabalho visando o incentivo da prática de atividades físicas entre os professores trazendo benefícios físicos e consequentemente melhora na qualidade de vida.

Entre professores que relataram nunca ou raramente terem tido atividade de lazer foi constatada a associação ao comprometimento do domínio Meio ambiente. $\mathrm{O}$ achado pode ser esperado uma vez que esse domínio contempla facetas que mensuram a participação em oportunidades de recreação ou lazer, recursos financeiros, entre outras. Ressalta-se a importância desse achado, uma vez que o lazer pode proporcionar divertimento e sensação de bem-estar ao indivíduo e quando não disponível pode comprometer a saúde mental, conforme estudo que verificou que o tempo insuficiente para a família e lazer teve associação significativa à síndrome de Burnout (Koga et al., 2015).

Os fatores relacionados aos distúrbios do sono como nunca/raramente acordar descansado e acordar durante a noite estiveram associados respectivamente aos domínios Meio ambiente e Psicológico. Entre docentes universitários, aspectos psicológicos como a depressão, ansiedade, dor musculoesquelética e estresse cooperaram com a perda ou má qualidade do sono (Sanches et al., 2019). Além desses, os fatores ambientais como poucas atividades de lazer, entre outros, também influenciaram para uma menor qualidade de vida (Sanches et al., 2019). Na literatura, o sono é cada vez mais reconhecido como um importante fator de estilo de vida para a saúde (St-Onge et al., 2016), ou seja, a privação do sono interfere no bem estar físico e mental e pode levar a prejuízos nas relações sociais e interpessoais dos indivíduos, uma vez que o sono possui função reparadora, de conservação de energia, de proteção e imunológica (Neves, Macedo \& Gomes, 2017). Nota-se que as queixas de distúrbios do sono são comuns entre professores (Scheuch, Haufe \& Seibt, 2015). Portanto, o achado deste estudo confirma mais uma vez a influência de distúrbios do sono na QV e chama a atenção de profissionais da saúde para a investigação desse fator entre professores (Ferreira et al., 2010; Ming Ng Y, Voo P, Maakip, 2010).

Dos fatores relacionados à condição de saúde (Bloco 4), a ocorrência de Transtorno Mental Comum esteve significativamente associada ao comprometimento dos domínios Físico, Psicológico e Relações sociais. Esse achado se assemelha a outro estudo que encontrou todos os indicadores da síndrome de burnout associados com a qualidade de vida em todos os domínios, evidenciando que quanto maior o desgaste, menor a QV de professores (Tabeleão, Tomasi \& Neves, 2011). Isto pode ser considerado preocupante, pois relatos de doenças mentais e psicossomáticas são frequentemente encontradas entre professores (Scheuch, Haufe \& Seibt, 2015; Luz et al., 2019; Graup et al.,2020). Além disso, têm sido sustentado a ideia de que fatores psicossociais e depressão são preditores significativos de dor musculoesquelética (Ming, Voo \& Maakip, 2010) e de distúrbios de voz (Mota et al., 2019) entre professores.

As queixas de dores musculoesqueléticas são frequentemente reportadas por professores (Fernandes, Rocha \& Fagundes, 2011; Ceballos \& Santos, 2015) e outro estudo brasileiro também evidenciou o comprometimento na qualidade de vida de professores em decorrência da presença desses sintomas nos últimos 7 dias (Fernandes, Rocha \& Fagundes, 2011). No estudo em questão, as queixas de dores musculoesqueléticas estiveram significativamente associadas aos domínios Físico e Meio ambiente entre os que referiram sentir dor nos últimos 7 dias e no domínio Relações sociais entre os que tiveram queixas nos últimos 12 meses. Em todos os casos não há como estabelecer uma relação de causa e efeito dessa associação por se tratar de um estudo transversal, além disso, pelo fato de a experiência da dor ser um evento complexo e multifatorial outras investigações podem ser necessárias para compreender este evento. No entanto, é valida a sugestão de investimentos em saúde 
ocupacional para minimizar os relatos de dor, no sentido de haver um planejamento da estrutura física escolar, mobiliários e equipamentos adequados ergonomicamente aos professores, além do incentivo de estratégias que reduzam o sedentarismo e garantam espaços e horários para a prática de atividade física (Lopes et al., 2018).

Neste estudo, no bloco 4, a presença de distúrbio de voz foi fator associado ao comprometimento do domínio Meio ambiente. As características do ambiente de trabalho, como a quantidade de alunos por sala, exposição a pó de giz e microrganismos podem afetar a voz do professor (Birolim et al., 2019). O resultado encontrado neste estudo diverge de outro que envolveu professores de uma instituição pública federal de educação profissional e tecnológica, no qual verificou que embora os docentes tenham apresentado queixas vocais, elas não se refletiram na limitação da qualidade de vida (Lopes et al., 2018). Entre os estudos, diferenças quanto ao sexo, idade dos alunos entre os níveis de ensino e tipo de rede mantenedora da escola justificariam esse achado, contudo, destaca-se que no ambiente de trabalho em escolas públicas municipais, como do presente estudo, diferem de uma instituição federal. Nesse aspecto, faz-se necessário ponderar sobre as diferenças entre as condições de trabalho de professores que atuam em escolas municipais, estaduais e federais, principalmente quanto à missão, gestão administrativa e infraestrutura, pois piores condições socioeconômicas e de infraestrutura geralmente são encontradas nas escolas municipais brasileiras (Assunção \& Oliveira, 2009).

Finalmente, faz-se necessário destacar as limitações e avanços deste estudo. Ao fato de ser um estudo de delineamento transversal, o seu resultado não possibilita estabelecer uma relação de causa e efeito e se limita a explorar apenas as associações entre as variáveis. O efeito do trabalhador sadio deve ser considerado, visto que os professores afastados no período da coleta por problemas de saúde não participaram, e desse modo àqueles com comprometimentos na saúde e que poderiam ter piores níveis de qualidade de vida não se incluíram no estudo.

Quanto aos avanços, o estudo tem relevância por apresentar amostra aleatória e representativa de uma população de professores. Bem como, o estudo inova ao agregar nas análises os testes estatísticos do tipo de regressão hierarquizada, no qual incluem uma ampla gama de fatores investigados e simultaneamente trabalham com a investigação de distúrbios osteomuscular, mental e vocal, que são os três agravos que mais afetam a saúde dos professores.

Diante do exposto, se faz necessário maior atenção aos fatores que comprometem a QV e, por consequência, afetam a saúde dos professores. A implementação de linhas de cuidado aos trabalhadores, com serviços organizados em redes para a promoção, tratamento e reabilitação da saúde, pode ser um componente fundamental para a integralidade do cuidado (Brasil, 2018). Assim, os achados deste estudo podem contribuir com políticas e programas de saúde individual e coletiva ao subsidiar a identificação de elementos importantes nos contextos socioeconômicos, de trabalho e saúde dos professores.

\section{Conclusão}

Comprometeram os domínios de qualidade de vida alguns fatores relacionados às características sociodemográficas, do trabalho docente, de hábitos de vida e sono, transtorno mental, queixa osteomuscular e distúrbios de voz. A associação do mesmo fator a um ou mais domínios avaliados reforça a inter-relação existente entre fatores que afetam a QV. Desse modo, foram fatores associados ao comprometimento do domínio Físico trabalhar uma carga horária $\geq 40$ horas, a falta de tempo para realizar as atividades na escola, a percepção de que o trabalho interfere na saúde, não realizar atividade física/esportes, a presença de sintomas de transtorno mental comum (TMC) e queixas osteomusculares. No domínio Psicológico, estavam associados fatores como à manifestação de que o trabalho é repetitivo; falta de tempo para realizar as atividades na escola; percepção de que o trabalho interfere na saúde; dificuldades quanto ao sono; e a presença de TMC.

O comprometimento do domínio Relações sociais estive associado a fatores como, viver sem o cônjuge, lecionar por 10-19 anos, perceber no ambiente de trabalho da escola a ocorrência de ruído, realizar esforço físico intenso no trabalho, ocorrência de TMC e queixa osteomuscular. No domínio Meio ambiente, estavam associados fatores como o tempo de 
deslocamento da casa para trabalho maior e igual a 31minutos, à percepção de que o trabalho interfere na saúde, falta de atividades de lazer, não acordar descansado, a presença do distúrbio de voz e ocorrência de queixas osteomusculares.

\section{Referências}

Assunção, A. Á., \& Oliveira, D. A. (2009). Intensificação do trabalho e saúde dos professores. Educação \& Sociedade, 30(107), $349-372$.

Birolim, M. M., Mesas, A. E., González, A. D., Santos, H. G., Haddad, M. C. F. L., \& Andrade, S. M. (2019). Job strain among teachers: associations with occupational factorsaccording to social support. Ciência \& Saúde Coletiva, 24(4),1255-64.

Bolfarine, H., \& Bussab, W. O. (2005). Elementos de amostragem. Editora Edgar Blücher.

Brasil (2018), Departamento de Vigilância em Saúde Ambiental e Saúde do Trabalhador. Distúrbio de Voz Relacionado ao Trabalho - DVRT. Brasília: Ministério da Saúde, 2018.

Ceballos, A. G. C., \& Santos, G. B. (2015). Factors associated with musculoskeletal pain among teachers: sociodemographics aspects, general health and wellbeing at work. Revista Brasileira epidemiologia, 18(3),702-715.

Cortez, P. F., Souza, M. V. R., Amaral, L. O., \& Silva, L. C. A. (2017). A saúde docente no trabalho: apontamentos a partir da literatura recente. Cadernos Saúde Coletiva, 25(1), 113-122.

Davoglio, T. R., Lettnin, C. C., \& Baldissera, C. G. (2015). Avaliação da qualidade de vida em docentes brasileiros: uma revisão sistemática. Pro-Posições, $26(3), 145-166$.

Delcor, N. S., Araújo, T. M., Reis, E. J. F. B., Porto, L. A., Carvalho, F. M., Silva, M. O., et al (2004). Condições de trabalho e saúde dos professores da rede particular de ensino de Vitória da Conquista, Bahia, Brasil. Cadernos de Saúde Pública, 20(1), 187-196.

Dias, D. F., Loch, M. R., González, A. D., Andrade, S. M., \& Mesas, A. E. (2017). Insufficient free-time physical activity and occupational factors in Brazilian public school teachers. Revista Saúde Publica, 51, 68.

Espinosa, M. M., Oliveira, N. L., Rodrigues, D. C., Alves, B. M. M., \& Marcon, S. R. (2019). Comparison of regression models between quantitative and categorical variables in the studies on the quality of life of the elderly. Ciência e Natura, Santa Maria, 41 (e26): 01-13.

Fernandes, M. H., da Rocha, V. M., \& Fagundes, A. A. (2011). Impacto da sintomatologia osteomuscular na qualidade de vida de professores. Revista Brasileira Epidemiologia,14(2), 276-84.

Ferreira, L. P., Latorre, M. R. D. O., Giannini, S. P. P., Ghirardi, A. C. A. M., Karmann, D. F., Silva, E. E., \& Figueira, S. (2010). Influence of Abusive Vocal Habits, Hydration, Mastication, and Sleep in the Occurrence of Vocal Symptoms in Teachers. Journal of Voice, $24,86-92$.

Fillis, M. M. A., Andrade, S. M., González, A. D., Melanda, F. N., \& Mesas, A. E. (2016). Frequência de problemas vocais autorreferidos e fatores ocupacionais associados em professores da educação básica de Londrina, Paraná, Brasil. Cadernos Saúde Pública, $32(1)$, e00026015.

Folle, A., \& Farias, G (2012). Nível de qualidade de vida e de atividade física de professores de escolas públicas estaduais da cidade de Palhoça (SC). Revista Mackenzie de Educação Física e Esporte, 11(1),11-21.

Gabani, F. L., González, A. D., Mesas, A. E., \& Andrade, S. M. (2018). The most uncomfortable chronic pain in primary school teachers: differential between different body regions. Brazilian Journal of Pain, 1(2),151-7.

Ghirardi, A. C. A., Ferreira, L. P.; Giannini, S. P. P.; \& Latorre, M. R. D. O. (2013). Screening Index for Voice Disorder (SIVD): Development and Validation. J. Voice, 27(2), 195-200.

Giannini, S. P. P., Latorre, M. D. R. D. D., \& Ferreira, L. P (2016). Condition of Vocal Production-Teacher questionnaire: comparison of responses on Likert scale and visual analog scale. CoDAS, 28(1), 53-8.

Gonçalves, D. M., Stein, A. T., \& Kapczinski, F (2008). Avaliação de desempenho do Self-Reporting Questionnaire como instrumento de rastreamento psiquiátrico: um estudo comparativo com o Structured Clinical Interview for DSM-IV-TR, 2007. Cad Saúde Pública, 24(2), 380-90.

Graup, S., Aguiar, A. H. R., Teixeira, A. M., Bueno, L. R., Ribas, N. Y. P. C., \& Lara, S. (2020). Prevalência de transtornos mentais e fatores associados em professores de Educação Física. Research, Society and Development, 9(8), e290985060. http://dx.doi.org/10.33448/rsd-v9i8.5060

Instituto Nacional de Estudos de Pesquisas Educacionais Anísio Teixeira (INEP)(2018). Sinopses Estatísticas da Educação Básica. http://inep.gov.br/web/guest/sinopses-estatisticas-da-educacao-basica

Koga, G. K. C., Melanda, F. N., Santos, H. G., Sant'Anna, F. L., González, A. D., Mesas, A. E., et al (2015). Fatores associados a piores níveis na escala de Burnout em professores da educação básica. Cadernos saúde coletiva, 23(3), 268-275.

Lopes, M. C. L. A., Fagundes, S. N., Mousinho, K. C., Correia, M. G. C., Ribeiro, C. M. B., \& Vanderlei, A. D. (2018). Factors associated with vocal health and quality of life in teachers/professors. Revista CEFAC, 20(4), 515-531.

Luz, J. G., Pessa, S. L. R., Luz, R. P., \& Schenatto, F. J. A. (2019). Implicações do ambiente, condições e organização do trabalho na saúde do professor: uma revisão sistemática. Ciência saúde coletiva, 24(12), 4621-4632.

Minayo, M. C. S., Hartz, Z. M. A., \& Buss, P. M. (2000). Qualidade de vida e saúde: um debate necessário. Ciência saúde coletiva, $5(1)$, 7-18. 
Ming Ng, Y., Voo, P., \& Maakip, I. (2010). Psychosocial factors, depression, and musculoskeletal disorders among teachers. BMC Public Health, $19,234$.

Mota, A. F. B., Giannini, S. P. P., Oliveira, I. B., Paparelli, R., Carmo, R. D., \& Ferreira, L. P. (2019). Voice Disorder and Burnout Syndrome in Teachers. Journal of voice, 33(4), 581.e7-581.e16.

Neves, G. S. M. L., Macedo, P. \& Gomes, M. M. (2017). Transtornos do sono: atualização (1/2). Revista Brasileira Neurologia, 53(3), 19-30. http://docs.bvsalud.org/biblioref/2017/12/876873/rbn-533-3-transtornos-do-sono-1-2.pdf

Neves, M. Y. R., Brito, J. C., \& Muniz, H. P. (2019). Women schoolteachers' health, gender issues, and work in elementary education. Cadernos de Saúde Pública, 35 (Sup. 1:e00189617).

Pereira, E. F., Teixeira, C. S., Andrade, R. D., \& Silva-Lopes, A. (2014). O trabalho docente e a qualidade de vida dos professores na educação básica. Revista Salud Publica, 16(2), 221-231.

Pereira, É. F., Teixeira, C. S., \& Lopes, A. S. (2013). Qualidade de vida de professores de educação básica do município de Florianópolis, SC, Brasil. Ciência saúde coletiva, 18(7), 1963-1970.

Pero, V., \& Stefanelli, V. (2015). A questão da mobilidade urbana nas metrópoles brasileiras. Revista de Economia Contemporânea, 19(3), 366-402.

Pimentel, B. N., Fedosse, E., Rodrigues, N. G. S., Cruz, K. S., \& Santos-Filha, V. A. V. (2016). Perception of noise, hearing health and quality of life of public school teachers. Audiology - Communication Research, 21:e1740.

Pinheiro, F. A., Tróccoli, B. T., \& Carvalho, C. V. (2002). Validação do Questionário Nórdico de Sintomas Osteomusculares como medida de morbidade. Revista Saúde Pública, 36(3), 307-312.

Sanchez, H. M., Sanchez, E. G. M., Barbosa, M. A., Guimarães, E. C., \& Porto, C. C. (2019). Impact of health on quality of life and quality of working life of university teachers from different areas of knowledge. Ciência saúde coletiva, 24(11), 4111-4122.

Santos, M. N., \& Marques, A. C. (2013). Condições de saúde, estilo de vida e características de trabalho de professores de uma cidade do sul do Brasil. Ciência saúde coletiva, 18(3), 837-846.

Scheuch, K., Haufe, E., \& Seibt, R. (2015). Teachers' Health. Dtsch Arztebl Int., 112(20),347-56.

Silva, J. P., \& Fischer, F. M. (2020). Multiform invasion of life by work among basic education teachers and repercussions on health. Revista de Saúde Pública, 54, 3 .

St-Onge, M. P., Grandner, M. A., Brown, D., Conroy, M. B., Jean-Louis, G., Coons, M., et al (2016). Sleep duration and quality: impact on lifestyle behaviors and cardiometabolic health: a scientific statement from the American Heart Association. Circulation, 134(18), e367-86.

Tabeleão, V. P., Tomasi, E., \& Neves, S. F. (2011). Qualidade de vida e esgotamento profissional entre docentes da rede pública de Ensino Médio e Fundamental no Sul do Brasil. Cadernos Saúde Pública, 27(12), 2401-2408.

Tavares, D. D. F., de Oliveira, R. A. R., Mota Júnior, R. J., Oliveira, C. E. P., \& Marins, J. C. B. (2015). Quality of life of basic education teachers of public schools. Revista Brasileira em Promoção a Saúde, 28(2), 191-197.

Tostes, M. V., Albuquerque, G. S. C., Silva, M. J. S., \& Petterle, R. R. (2018). Sofrimento mental de professores do ensino público. Saúde em debate, 42(116), 87-99.

Victora, C. G., Huttly, S. R., Fuchs, S. C., \& Olinto, M. T. (1997). The role of conceptual frameworks in epidemiological analysis: a hierarchical approach. International Journal of Epidemiology, 26(1), 224-227.

World Health Organization. (1998; 2012). Programme on mental health: WHOQOL user manual, 2012 revision. http://www.who.int/iris/handle/10665/77932

Zavidovique, L., Gilbert, F., \& Vercambre-Jacquot, M. N. (2018). Bien-être au travail et qualité de vie des enseignants : quelles différences selon l'ancienneté ? Archives des Maladies Professionnelles et de l'Environnement, 79:105-119. 